\title{
Hypoalbuminaemia is associated with mortality in patients undergoing cytoreductive nephrectomy
}

\author{
Anthony T. Corcoran*, Samuel D. Kaffenberger ${ }^{\dagger}$, Peter E. Clark ${ }^{\dagger}$, John Walton ${ }^{\ddagger}$, \\ Elizabeth Handorf§, Zack Piotrowski, Jeffery J. Tomaszewski, Serge Ginzburg", \\ Reza Mehrazin ${ }^{\ddagger}$, Elizabeth Plimack**, David Y.T. Chen ${ }^{\ddagger}$, Marc C. Smaldone ${ }^{\ddagger}$, \\ Robert G. Uzzo $\ddagger$, Todd M. Morgan ${ }^{\dagger \dagger}$ and Alexander Kutikov ${ }^{\ddagger}$
}

\begin{abstract}
*Department of Urology, SUNY Stony Brook Medical Center, Stony Brook, NY, " ${ }^{*}$ Division of Urologic Oncology, §Biostatistics \& Bioinformatics Facility, * *Medical Oncology, Fox Chase Cancer Center-Temple University Health System, Philadelphia, PA, 'Department of Urology, Vanderbilt University School of Medicine, Nashville, TN, "Department of Urology, Einstein Healthcare Network/Urologic Institute of Southeastern Pennsylvania, Philadelphia, PA, and ${ }^{+t}$ Department of Urology, University of Michigan School of Medicine, Ann Arbor, MI, USA
\end{abstract}

\section{Objective}

To evaluate whether poor nutrition is associated with mortality in patients undergoing cytoreductive nephrectomy (CN) for metastatic renal cell carcinoma (mRCC).

\section{Patients and Methods}

A multi-institutional review of prospective databases identified 246 patients meeting inclusion criteria who underwent CN for mRCC from 1993 to 2012. Nutritional markers evaluated were: body mass index $<18.5 \mathrm{~kg} / \mathrm{m}^{2}$, serum albumin $<3.5 \mathrm{~g} / \mathrm{dL}$, or preoperative weight loss of $\geq 5 \%$ of body weight. Primary outcomes were overall (OS) and disease-specific survival (DSS). Secondary outcome was 'early mortality' defined as death at $\leq 6$ months of surgery. Survival curves were estimated using the Kaplan-Meier product-limit method and multivariate analysis using logistic regression was used to test associations between nutritional markers and survival outcomes.

\section{Results}

In all, 119 patients (median follow-up 17 months) were categorised as having any abnormal nutrition parameter
(48\%). Hypoalbuminaemia was the only independent predictor of OS and DSS (OS: median 8 vs 23 months, $P<0.001$; DSS: 11 vs 33 months, $P<0.001)$. On multivariate analysis, hypoalbuminaemia remained a significant predictor of death for both overall [hazard ratio (HR) 2, 95\% confidence interval (CI) $1.4-2.8 ; P<0.001)$ and disease-specific mortality (HR 2.2, 95\% CI 1.4-3.3; $P<0.001$ ). Hypoalbuminaemia was also associated with early mortality (overall: $P<0.001$ and disease specific: $P=0.002$ ).

\section{Conclusion}

Patients with mRCC and hypoalbuminaemia undergoing CN have decreased OS and CSS, and increased risk of all-cause and disease-specific early mortality. As such, serum albumin may help risk stratify patients selected as candidates for CN. Furthermore, future work should evaluate whether nutritional depletion is a modifiable risk factor.

\section{Keywords}

metastatic renal cell carcinoma, cytoreductive nephrectomy, nutritional status, systemic therapy, survival

\section{Introduction}

Before 2001, the role of nephrectomy in the setting of mRCC was largely undefined, and radical surgery was offered chiefly for palliative purposes. However, two prospective randomised trials showed that cytoreductive nephrectomy $(\mathrm{CN})$ imparts a survival advantage for patients with mRCC who then undergo immunotherapy when compared with patients who receive interferon- $\alpha$ alone [1-3]. Despite the advent of targeted therapy and loss of enthusiasm for interferon- $\alpha$ [4-6], CN continues to be a mainstay therapeutic option for patients with mRCC [7].

$\mathrm{CN}$ may not benefit all patients and in fact may be harmful to a subset of those who are destined to either have major complications from surgery or to exhibit rapid disease progression [8]. In fact, such patients may miss an opportunity to receive potentially life-extending systemic targeted therapy 
[8-10]. Thus, preoperative risk stratification to identify high-risk cohorts is extremely desirable but has proven to be clinically challenging $[11,12]$.

Poor nutritional status is a known predictor of survival in various surgical settings [13-16]. Recently a robust association between poor nutritional status and postoperative mortality in patients with locoregional RCC was reported [17]. However, identification of a relationship between nutritional status and survival after $\mathrm{CN}$ is yet to be fully defined [18]. As such, the purpose of the present study was to evaluate the association between preoperative nutritional deficiency and poor survival outcomes after CN.

\section{Patients and Methods}

After individual Institutional Review Board approval for the creation and analysis of two prospectively maintained databases (Fox Chase Cancer Center and Vanderbilt University Medical Center), patients with mRCC treated with CN from 1993 to 2012 were identified. Metastatic disease was confirmed through biopsy or radiographic imaging and was characterised as involving lung, bone, liver, brain, or other sites. Patients with insufficient staging records, pathological findings of a non-renal cell malignancy, history of previous treatment for locoregional disease, and those without appropriate nutritional status data were excluded from the study. Surgery was performed via open, laparoscopic or robotic approaches. Treating physicians determined follow-up schedules based on their personal and institutional clinical practice. Primary outcomes were overall (OS) and disease-specific survival (DSS). Using data from both the Southwestern Oncology Group 8949 and European Organization for Research and Treatment of Cancer 30947 randomised trials first evaluating the use of $\mathrm{CN}$ in $\mathrm{mRCC}$, the OS advantage in the $\mathrm{CN}$ group was 6 months [2]. Therefore, a secondary outcome was 'early mortality' defined as death at $\leq 6$ months of surgery to evaluate if any net benefit was obtained from CN. Follow-up was determined from time of surgery to time of last contact with the patient or date of death. Death dates were confirmed by death certificate. Overall mortality vs disease-specific mortality was determined by reconciliation of data from death certificates, National Death Index, institutional tumour registries, and physician documentation.

Although most of the data were collected prospectively, medical records were reviewed retrospectively to obtain missing and desired data points. Nutritional parameters assessed included serum albumin, body mass index (BMI) and percentage weight loss within the 6 months before surgery with thresholds for abnormal parameters of: serum albumin $<3.5 \mathrm{~g} / \mathrm{dL}, \mathrm{BMI}<18.5 \mathrm{~kg} / \mathrm{m}^{2}$, or unintentional weight loss of $\geq 5 \%$ of total body weight. Consistent with prior reports, survival outcomes were also evaluated between patients using a composite classification for nutritional deficiency [ $\geq$ one parameter abnormal: serum albumin $<3.5 \mathrm{~g} / \mathrm{dL}$ (hypoalbuminaemia), BMI $<18.5 \mathrm{~kg} / \mathrm{m}^{2}$, or unintentional weight loss of $\geq 5 \%$ of total body weight] compared with nutritionally replete patients (all parameters normal) [17]. Each nutritional parameter was also evaluated individually. Anaemia was defined as serum haematocrit of $<41 \%$ for men and $<36 \%$ for women. Symptoms were defined as local or metastatic if pain or discomfort at site was attributable to RCC or systemic only if constitutional symptoms were present. Preoperative renal function was assessed with estimated GFR and chronic kidney disease staged according to the National Kidney Foundation staging system [19]. Pathological characteristics included tumour stage, histological subtype, Fuhrman nuclear grade, presence of sarcomatoid variant and nodal status. Pathological classification of tumours was carried out in accordance with the 2010 American Joint Committee on Cancer classification system [20].

Association between nutritional status and patient and disease characteristics was assessed using Fisher's exact or chi-square tests for categorical variables. Kaplan-Meier survival curves were constructed from univariate analyses for DSS and OS after $\mathrm{CN}$, and significance was assessed using the log-rank test. In multivariate survival analyses, Cox proportional hazards models for OS and DSS were constructed adjusting for factors significant and approaching significance on univariate analysis. Statistical analysis was carried out using STATA software (version 12.1).

\section{Results}

In all, 275 consecutive patients with mRCC treated with CN from 1993 to 2012 were identified from two prospectively maintained institutional databases. Of these 246 patients met study inclusion criteria with a median (interquartile range, IQR) age of 60 (52-67) years. The median (IQR) follow-up was 17 (5-31) months. The patients' demographic characteristics are listed in Table 1. The majority of patients were men $(71.5 \%)$, had a preoperative Eastern Cooperative Oncology Group Performance Status of 0-1 (90\%), and were treated with open radical nephrectomy $(74 \%)$. Lung metastases were present in $66 \%$ of patients with $17.5 \%$ of patients having lung as the only site of metastasis. Bone metastases were present in $36 \%$ of patients, while liver and brain metastases were present in $17.9 \%$ and $5.3 \%$, respectively. In all, $57 \%$ of patients had multiple sites of metastatic involvement.

Summarised in Table 2, of our total cohort, hypoalbuminaemia was present in $25 \%(62 / 246)$ while nearly half $(48.4 \%)$ met at least one criteria for nutritional deficiency. Predictors of OS and DSS on univariate analysis are summarised in Tables 3 and 4. In an initial multivariable model, patients meeting the 
Table 1 Patient demographics.

\begin{tabular}{|c|c|c|c|c|}
\hline Variable & Total, $N(\%)$ & Low serum albumin, $N(\%)$ & Normal serum albumin, $N(\%)$ & $\mathbf{P}$ \\
\hline All patients & $246(100.0)$ & $62(25.2)$ & $184(74.8)$ & \\
\hline Men & $176(71.5)$ & $44(71.0)$ & $132(71.7)$ & 1 \\
\hline \multicolumn{5}{|l|}{ Age at $\mathrm{CN}$, years: } \\
\hline$\leq 50$ & $51(20.7)$ & $17(27.4)$ & $34(18.5)$ & \\
\hline $51-60$ & $73(29.7)$ & $16(25.8)$ & $57(31.0)$ & \\
\hline $61-70$ & $84(34.1)$ & $20(32.3)$ & $64(34.8)$ & \\
\hline $71-80$ & $33(13.4)$ & $7(11.3)$ & $26(14.1)$ & \\
\hline$>80$ & $5(2.0)$ & $2(3.2)$ & $3(1.6)$ & 0.504 \\
\hline \multicolumn{5}{|l|}{ Race: } \\
\hline Black & $17(6.9)$ & $5(8.1)$ & $12(6.5)$ & \\
\hline Other & $229(93.1)$ & $57(91.9)$ & $172(93.5)$ & 0.772 \\
\hline \multicolumn{5}{|l|}{ Year of CN: } \\
\hline 1993-1995 & $3(1.2)$ & 0 & $3(1.6)$ & \\
\hline $1996-2000$ & $13(5.3)$ & $6(9.7)$ & $7(3.8)$ & \\
\hline 2001-2005 & $101(41.1)$ & $31(50.0)$ & $70(38.0)$ & \\
\hline 2006-2010 & $113(45.9)$ & $23(37.1)$ & $90(48.9)$ & \\
\hline 2011-2012 & $16(6.5)$ & $2(3.2)$ & $14(7.6)$ & 0.093 \\
\hline \multicolumn{5}{|l|}{ ECOG PS: } \\
\hline $0-1$ & $216(87.8)$ & $51(82.3)$ & $165(89.7)$ & \\
\hline$>1$ & $24(9.8)$ & $9(14.5)$ & $15(8.2)$ & \\
\hline NS & $6(2.4)$ & $2(3.2)$ & $4(2.2)$ & 0.21 \\
\hline \multicolumn{5}{|l|}{ CCI: } \\
\hline$<7$ & $123(50.0)$ & $35(56.5)$ & $88(47.8)$ & \\
\hline $7-9$ & $106(43.1)$ & $21(33.9)$ & $85(46.2)$ & \\
\hline$>9$ & $17(6.9)$ & $6(9.7)$ & $11(6.0)$ & 0.185 \\
\hline \multicolumn{5}{|l|}{ Symptoms: } \\
\hline None & $23(9.3)$ & $3(4.8)$ & $20(10.9)$ & \\
\hline Local only & $63(25.6)$ & $17(27.4)$ & $46(25.0)$ & \\
\hline Metastatic only & $65(26.4)$ & $5(8.1)$ & $60(32.6)$ & \\
\hline Systemic only & 34 (13.8) & $15(24.2)$ & $19(10.3)$ & \\
\hline$>1$ & $61(24.8)$ & $22(35.5)$ & $39(21.2)$ & $<0.001$ \\
\hline \multicolumn{5}{|l|}{ Renal insufficiency: } \\
\hline \multirow{2}{*}{\multicolumn{5}{|c|}{ Anaemia: }} \\
\hline & & & & \\
\hline Yes & $155(63.0)$ & $55(88.7)$ & $100(54.3)$ & $<0.001$ \\
\hline \multicolumn{5}{|l|}{ Nephrectomy: } \\
\hline Open & $182(74.0)$ & $46(74.2)$ & $136(73.9)$ & \\
\hline Laparoscopic & $63(25.6)$ & $16(25.8)$ & 47 (25.5) & \\
\hline Robotic & $1(0.4)$ & $1(1.6)$ & 0 & 1 \\
\hline Intraoperative tran & & & & \\
\hline Yes & $90(36.6)$ & $36(58.1)$ & $54(29.3)$ & $<0.001$ \\
\hline Site of metastasis: & & & & \\
\hline Lung & $163(66.3)$ & $52(83.9)$ & $111(60.3)$ & 0.001 \\
\hline Bone & $89(36.2)$ & $15(24.2)$ & $74(40.2)$ & 0.032 \\
\hline Liver & $44(17.9)$ & $17(27.4)$ & $27(14.7)$ & 0.034 \\
\hline Brain & $13(5.3)$ & $4(6.5)$ & $9(4.9)$ & 0.74 \\
\hline NOS & $123(50.0)$ & $31(50.0)$ & $92(50.0)$ & 0.99 \\
\hline No. metastatic sites & & & & \\
\hline 1 & $105(42.7)$ & $23(37.1)$ & $82(44.6)$ & \\
\hline 2 & $100(40.7)$ & $23(37.1)$ & 77 (41.8) & \\
\hline 3 & $38(15.4)$ & $14(22.6)$ & $24(13.0)$ & \\
\hline$>3$ & $3(1.2)$ & $2(3.2)$ & $1(0.5)$ & 0.09 \\
\hline Pathological tumou & & & & \\
\hline$<\mathrm{pT} 3 \mathrm{~b}$ & $174(70.7)$ & $36(58.1)$ & $138(75.0)$ & 0.036 \\
\hline pТ3b & $32(13.0)$ & $11(17.7)$ & $21(11.4)$ & \\
\hline$>\mathrm{pT} 3 \mathrm{~b}$ & $40(16.3)$ & $15(24.2)$ & $25(13.6)$ & \\
\hline Pathological nodal & & & & \\
\hline No/NX & $173(70.3)$ & $41(66.1)$ & $132(71.7)$ & \\
\hline $\mathrm{N}+$ & $73(29.7)$ & $21(33.9)$ & $52(28.3)$ & 0.424 \\
\hline Fuhrman grade: & & & & \\
\hline I-II & $40(16.3)$ & $3(4.8)$ & $37(20.1)$ & \\
\hline III-IV & $200(81.3)$ & $57(91.9)$ & $143(77.7)$ & \\
\hline NS & $6(2.4)$ & $2(3.2)$ & $4(2.2)$ & 0.009 \\
\hline Histology: & & & & \\
\hline Clear cell & $199(80.9)$ & $50(80.6)$ & $149(81.0)$ & \\
\hline Non-clear cell & $47(19.1)$ & $12(19.4)$ & $35(19.0)$ & \\
\hline Papillary & $16(6.5)$ & $1(1.6)$ & $15(8.2)$ & \\
\hline Mixed & $9(3.7)$ & $2(3.2)$ & $7(3.8)$ & \\
\hline Unclassified & $8(3.3)$ & $4(6.5)$ & $4(2.2)$ & \\
\hline Collecting duct & $8(3.3)$ & $1(1.6)$ & $7(3.8)$ & \\
\hline Sarcomatoid & $5(2.0)$ & $3(4.8)$ & $2(1.1)$ & \\
\hline Medullary & $1(0.4)$ & $1(1.6)$ & 0 & 0.002 \\
\hline Sarcomatoid comp & & & & \\
\hline Yes & $59(24.0)$ & $22(35.5)$ & $37(20.1)$ & 0.017 \\
\hline
\end{tabular}

CCI, Charlson comorbidity index; ECOG PS, Eastern Cooperative Oncology Group Performance Status; NOS, not otherwise specified. 
Table 2 Patient distribution of nutritional status.

\begin{tabular}{lc}
\hline Parameter $(\boldsymbol{N}=\mathbf{2 4 6})$ & No. patients (\%) \\
\hline Serum albumin, g/dL: & \\
$\quad<3.5$ & $62(25)$ \\
$>3.5$ & $153(62)$ \\
BMI, $\mathrm{kg} / \mathrm{m}^{2}:$ & $1(0.4)$ \\
$\quad<18.5$ & $224(91)$ \\
$>18.5$ & \\
Weight loss, \%: & $85(35)$ \\
$\geq 5$ & $160(65)$ \\
$<5$ & $119(48)$ \\
Composite nutrition status: & $127(52)$ \\
Nutritionally deficient & \\
Nutritionally replete & $90(76)$ \\
Number of abnormal parameters: & $29(24)$ \\
1 & 0 \\
2 & \\
3 & \\
\hline
\end{tabular}

Table 3 Cox univariate and multivariate regression analysis of OS.

\begin{tabular}{|c|c|c|c|c|c|c|}
\hline & \multicolumn{3}{|c|}{ Univariable } & \multicolumn{3}{|c|}{ Multivariable } \\
\hline & HR & $95 \% \mathrm{CI}$ & $\boldsymbol{P}$ & HR & $95 \% \mathrm{CI}$ & $\boldsymbol{P}$ \\
\hline Low serum albumin & 2.35 & $1.71-3.23$ & $<0.001$ & 1.91 & $1.31-2.78$ & $<0.001$ \\
\hline Weight loss $>5 \%$ & 1.48 & $1.10-2.00$ & 0.01 & 1.30 & $0.92-1.84$ & 0.142 \\
\hline Age & 1.00 & $0.99-1.01$ & 0.971 & 1.01 & $1.00-1.02$ & 0.203 \\
\hline $\mathrm{CCI}>10$ & 2.15 & $0.79-5.80$ & 0.132 & 1.32 & $0.43-4.02$ & 0.631 \\
\hline ECOG PS >1 & 1.19 & $0.89-1.60$ & 0.233 & 1.22 & $0.89-1.67$ & 0.215 \\
\hline Anaemia & 1.51 & $1.11-2.05$ & 0.009 & 1.02 & $0.71-1.46$ & 0.906 \\
\hline $\begin{array}{l}\text { Intraoperative } \\
\text { transfusion }\end{array}$ & 1.70 & $1.27-2.28$ & $<0.001$ & 1.24 & $0.86-1.80$ & 0.255 \\
\hline \multicolumn{6}{|l|}{ Symptoms: } & \\
\hline local only & 1.65 & $0.91-2.99$ & 0.099 & 1.39 & $0.75-2.58$ & 0.298 \\
\hline systemic only & 2.39 & $1.26-4.52$ & 0.007 & 1.33 & $0.67-2.64$ & 0.419 \\
\hline metastatic only & 1.21 & $0.66-2.20$ & 0.539 & 1.34 & $0.71-2.52$ & 0.364 \\
\hline$>1$ & 1.74 & $0.96-3.17$ & 0.07 & 1.18 & $0.62-2.25$ & 0.621 \\
\hline \multicolumn{7}{|l|}{ Metastatic sites: } \\
\hline $0-1$ & Referent & & & & & \\
\hline$>1$ & 1.95 & $1.44-2.64$ & $<0.001$ & 2.19 & $1.59-3.03$ & $<0.001$ \\
\hline \multicolumn{7}{|l|}{ Pathology: } \\
\hline$<\mathrm{T} 3 \mathrm{~b}$ & ref & & & & & \\
\hline T3b & 1.49 & $0.99-2.25$ & 0.058 & 1.11 & $0.70-1.77$ & 0.658 \\
\hline$>\mathrm{T} 3 \mathrm{~b}$ & 2.25 & $1.55-3.25$ & $<0.001$ & 1.29 & $0.83-2.00$ & 0.259 \\
\hline clear cell & 0.60 & $0.42-0.85$ & 0.004 & 0.53 & $0.34-0.82$ & 0.004 \\
\hline high grade & 2.16 & $1.44-3.25$ & $<0.001$ & 1.72 & $1.11-2.66$ & 0.015 \\
\hline $\begin{array}{l}\text { sarcomatoid } \\
\text { component }\end{array}$ & 1.80 & $0.67-4.87$ & 0.247 & 0.59 & $0.18-1.93$ & 0.38 \\
\hline node positive & 1.36 & $1.00-1.85$ & 0.051 & 0.86 & $0.60-1.23$ & 0.406 \\
\hline
\end{tabular}

CCI, Charlson comorbidity index; ECOG PS, Eastern Cooperative Oncology Group Performance Status.

criteria for nutritional deficiency using the composite score were at higher risk of overall [hazard ratio (HR) 1.70, 95\% CI $1.18-2.45 ; P=0.004$ ] and disease-specific (HR 1.81, 95\% CI $1.20-2.73 ; P=0.005)$ mortality. A second multivariate analysis using the individual nutritional deficiency parameters separately (Tables 3 and 4), controlling for factors significant and approaching significance on univariate analysis, only hypoalbuminaemia, polymetastases, non-clear cell histology,
Table 4 Cox univariate and multivariate regression analysis of DSS.

\begin{tabular}{|c|c|c|c|c|c|c|}
\hline & \multicolumn{3}{|c|}{ Univariable } & \multicolumn{3}{|c|}{ Multivariable } \\
\hline & HR & $95 \%$ CI & $\boldsymbol{P}$ & HR & $95 \% \mathrm{CI}$ & $\boldsymbol{P}$ \\
\hline Low serum albumin & 2.57 & $1.79-3.69$ & $<0.001$ & 2.07 & $1.35-3.18$ & 0.001 \\
\hline Weight loss $>5 \%$ & 1.75 & $1.25-2.45$ & 0.001 & 1.32 & $0.90-1.95$ & 0.158 \\
\hline Age & 1.00 & $0.98-1.01$ & 0.856 & 1.01 & $0.99-1.02$ & 0.262 \\
\hline $\mathrm{CCI}>10$ & 2.08 & $0.66-6.55$ & 0.211 & 1.51 & $0.40-5.63$ & 0.543 \\
\hline ECOG PS >1 & 1.37 & $0.98-1.93$ & 0.064 & 1.27 & $0.88-1.82$ & 0.195 \\
\hline Anaemia & 1.60 & $1.12-2.29$ & 0.01 & 1.21 & $0.73-1.67$ & 0.651 \\
\hline $\begin{array}{l}\text { Intraoperative } \\
\text { transfusion }\end{array}$ & 1.94 & $1.39-2.71$ & $<0.001$ & 1.21 & $0.79-1.84$ & 0.376 \\
\hline $\begin{array}{l}\text { Symptoms: } \\
\text { none }\end{array}$ & Referent & & & & & \\
\hline local only & 2.56 & $1.14-5.73$ & 0.022 & 1.77 & $0.77-4.07$ & 1.181 \\
\hline systemic only & 3.50 & $1.49-8.21$ & 0.004 & 1.59 & $0.64-3.94$ & 0.313 \\
\hline metastatic only & 2.05 & $0.91-4.59$ & 0.082 & 2.04 & $0.88-4.74$ & 0.096 \\
\hline$>1$ & 2.61 & $1.16-5.88$ & 0.021 & 1.85 & $0.78-4.37$ & 0.162 \\
\hline \multicolumn{7}{|l|}{ Metastatic sites: } \\
\hline $0-1$ & Referent & & & & & \\
\hline$>1$ & 1.86 & $1.31-2.62$ & $<0.001$ & 1.95 & $1.35-2.83$ & $<0.001$ \\
\hline \multicolumn{7}{|l|}{ Pathology: } \\
\hline$<\mathrm{T} 3 \mathrm{~b}$ & Referent & & & & & \\
\hline T3b & 1.59 & $0.99-2.54$ & 0.053 & 1.57 & $0.93-2.67$ & 0.093 \\
\hline$>\mathrm{T} 3 \mathrm{~b}$ & 2.49 & $1.64-3.77$ & $<0.001$ & 1.68 & $1.03-2.75$ & 0.038 \\
\hline clear cell & 0.63 & $0.42-0.95$ & 0.027 & 0.53 & $0.32-0.88$ & 0.015 \\
\hline high grade & 2.04 & $1.29-3.24$ & 0.002 & 1.58 & $0.96-2.62$ & 0.072 \\
\hline $\begin{array}{l}\text { sarcomatoid } \\
\text { component }\end{array}$ & 1.72 & $0.54-5.41$ & 0.357 & 0.53 & $0.13-2.21$ & 0.387 \\
\hline node positive & 1.42 & $1.00-2.02$ & 0.051 & 0.87 & $0.58-1.32$ & 0.519 \\
\hline
\end{tabular}

CCI, Charlson comorbidity index; ECOG PS, Eastern Cooperative Oncology Group Performance Status.

and high grade remained predictors of all-cause mortality (Table 3), while unintentional weight loss of $\geq 5 \%$ of total body weight was not significant. Only one patient had a BMI of $<18.5 \mathrm{~kg} / \mathrm{m}^{2}$, precluding evaluation in our multivariate model. Additionally, only serum albumin level, polymetastases, non-clear cell histology and stage $>\mathrm{T} 3 \mathrm{~b}$ were predictive of disease-specific mortality on multivariate analysis (Table 4).

Evaluating serum albumin levels independently, all-cause and disease-specific mortality was observed in $91.9 \%$ and $74.2 \%$ of patients with hypoalbuminaemia vs $69.6 \%$ and $51.1 \%$ in patients with normal levels, respectively. Kaplan-Meier survival analysis revealed significant differences in median OS ( 8 vs 23 months; $P<0.001$; Fig. 1 ) and DSS (11 vs 33 months; $P<0.001$; Fig. 2) in patients with low and normal serum albumin. Survival outcomes were analysed at 6 months to evaluate rates of early postoperative mortality. In multivariate analyses, adjusting for age, tumour grade, Charleston comorbidity index, presence of polymetastases, clear cell and sarcomatoid histologies, hypoalbuminaemia was significantly associated with all-cause (HR 1.91, 95\% CI 1.31-2.78; $P<$ 0.001 ) and disease-specific (HR 2.07, 95\% CI 1.35-3.18; $P<$ $0.001)$ mortality. At 6 months, both all-cause $[56.5 \%$ (95\% CI 43.3-67.7\%) vs $80.6 \%$ (95\% CI 74.1-85.7\%); $P<0.001]$ and disease-specific [62.6\% (95\% CI $48.9-73.6 \%)$ vs $84.3 \%$ (95\% CI 
Fig. 1 Kaplan-Meier analysis for OS in patients with low and normal serum albumin levels.

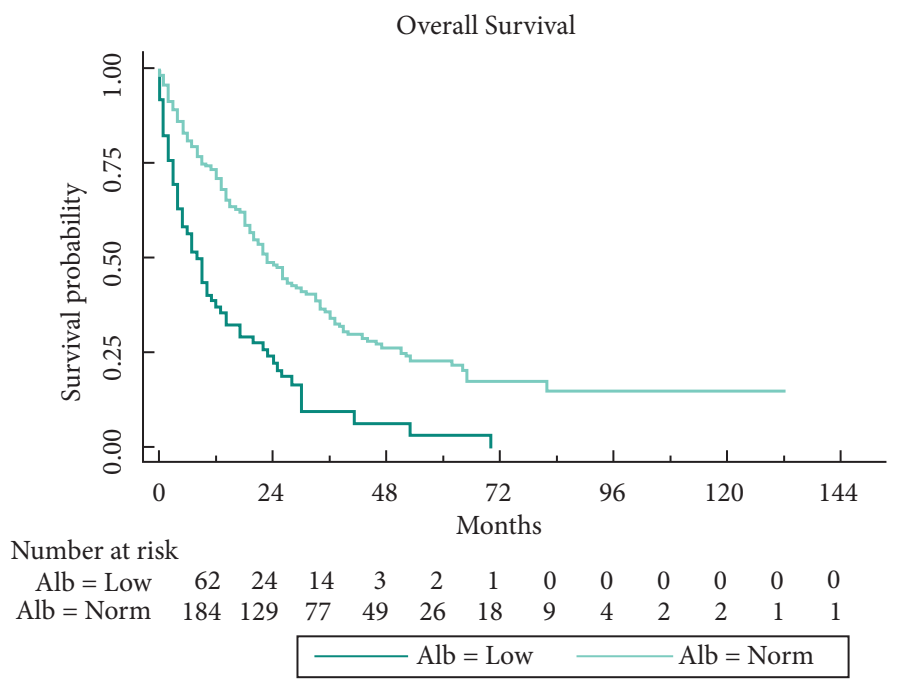

Fig. 2 Kaplan-Meier analysis for DSS in patients with low and normal serum albumin levels.

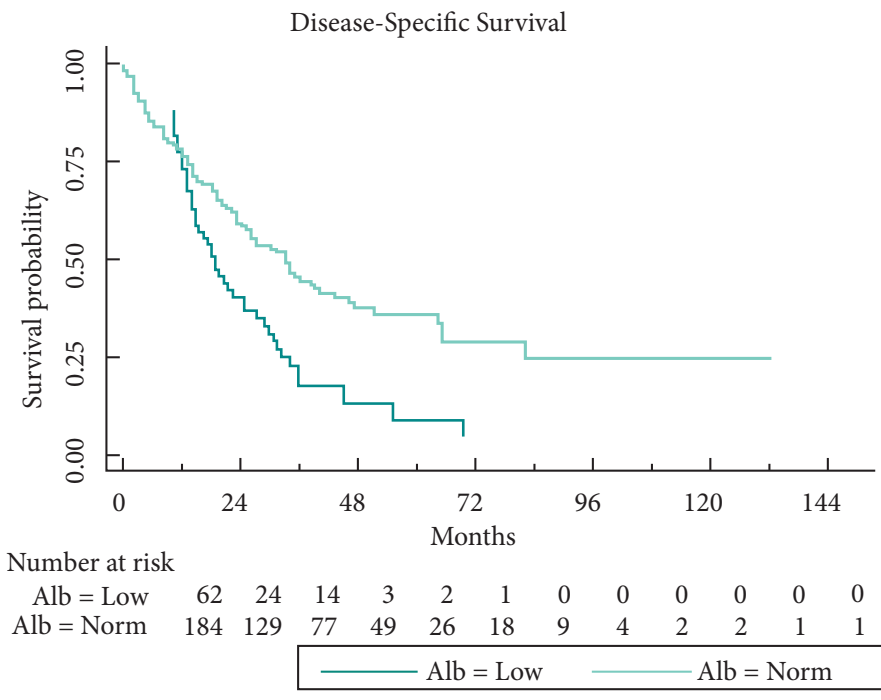

78.1-88.9\%); $P=0.002$ ) survival differed significantly for patients with hypoalbuminaemia compared with patients with normal albumin.

\section{Discussion}

In this large multi-institutional cohort of patients with mRCC, low preoperative serum albumin was an independent predictor of both overall and disease-specific mortality after $\mathrm{CN}$. Indeed, patients with hypoalbuminaemia had a $>50 \%$ reduction in OS and DSS when compared with patients with normal serum albumin levels and were at double the risk for early mortality.
These findings are highly significant, as the role of $\mathrm{CN}$ in the era of effective targeted therapy is yet to be fully defined [7]. Benefits of $\mathrm{CN}$ have only been shown in patients who uniformly received systemic therapy in the form of interferon- $\alpha$ after surgery, and clinical trials are underway to establish the survival role and timing of $\mathrm{CN}$ in the targeted therapy era [21,22]. In fact, $>30 \%$ of patients fail to receive systemic therapy after $\mathrm{CN}$ in contemporary cohorts $[8,10]$, as a large proportion of these patients do not receive systemic therapy due to rapid disease progression, perioperative morbidity and/or death $[8,10]$. Clearly, these patients are ill-served by cytoreductive surgery and unnecessarily incur surgical morbidity at the end of life, while foregoing an opportunity to receive potentially efficacious targeted therapy. As such, appropriate risk stratification of patients who present with metastatic disease is highly desirable to optimise the decision between $\mathrm{CN}$ or a trial of targeted therapy before surgery [7].

Progress in quantifying risk before $\mathrm{CN}$ recently has been reported $[9,18]$. Examining a cohort of patients undergoing $\mathrm{CN}$, Culp et al. [9], incorporated seven preoperative variables into a risk-group based model to identify those who benefit most from $\mathrm{CN}$. These and other predictors were then combined into pre- and post-operative multivariable models to predict DSS at 6 and 12 months after CN [18]. They then constructed a nomogram (area under the curve $=0.76$ ) to assess 6-month probability of death after cytoreductive surgery [18]. While the multivariable predictive models described may help identify patients with mRCC likely to benefit from $\mathrm{CN}$, our present study identifies serum albumin as the single most important predictor of OS and DSS in patients undergoing CN. Using a single clinical parameter rather than obtaining results from a nomogram may provide an advantage to practitioners for quick clinical application.

Serum albumin level represents an easily quantifiable and a potentially modifiable preoperative risk factor that has been extensively evaluated across various surgical disciplines, encompassing both benign and malignant disease states. In fact, robust data support the association between nutritional status, improved outcomes and decreased treatment costs [23-25]. Until recently, nutritional status has received only limited attention within urological oncology. One of the first studies examining nutritional status in patients with genitourinary malignancy evaluated patients undergoing radical cystectomy for localised bladder cancer [26]. Patients were classified as nutritionally deplete based on preoperative serum albumin levels, BMI or degree of perioperative weight loss and incurred worse perioperative (90-day) and overall mortality as compared with nutritionally replete patients. Similarly, these parameters were examined in patients with locoregional (non-metastatic) RCC with nutritionally deficient patients 
exhibiting higher 3-year overall and cancer-specific mortality [17].

Definitions of nutritional depletion vary [27]. Although nutritional deficiency metrics, such as the Nutritional Risk Screening tool (NRS), which assess nutritional status using weight loss, BMI, food intake, and disease severity, have a role in prospective studies, proponents of these metrics readily admit that quantification of food intake and disease severity are often qualitative and arbitrary. Furthermore, these metrics are impractical for retrospective assessments [27].

Recently, in urological literature, quantification of nutritional deficiency using serum albumin, BMI and weight loss has gained significant traction $[17,18,26]$. In fact this metric is generally readily quantifiable in routine clinical practice and was a strong predictor of poor outcomes in our cohort. However, our present data indicate that in patients with mRCC, serum albumin alone, independent of BMI or weight loss, was the component of the nutritional deficiency status associated with poor outcomes.

Previous reports show that serum albumin was abnormal in only $5 \%$ of patients with non-metastatic kidney cancer [17] and 6\% of those with localised bladder cancer [26]. In our present cohort $25 \%$ had abnormal serum albumin levels preoperatively. As such, our present data show that patients with mRCC who underwent surgical intervention had a five-fold increased risk of being malnourished than patients with localised urological malignancies $[17,26,27]$.

Our present data are consistent with previous reports showing that patients who have hypoalbuminaemia exhibit worse clinical outcomes than patients with normal albumin levels [9]. In fact patients with low serum albumin who underwent $\mathrm{CN}$ were at a 1.4-fold higher risk of 6-month all-cause and disease-specific mortality. These trends continued as the cohorts were followed long term, as patients with low albumin were $90 \%$ more likely to succumb to RCC and $80 \%$ more likely to meet an overall mortality end point at 17 months of median follow-up. In fact, patients with hypoalbuminaemia had a marked reduction in median OS (11 vs 26 months; $P<0.001$ ) and DSS (17 vs 46 months; $P<0.001)$ when compared with counterparts with normal levels.

Across various cancers, modulation of core factors, such as systemic inflammation and surgical stress, may be mediated via nutritional status, thus influencing the host's immune response. As such, nutritional repletion may harbour potential to enhance efficacy of existing therapies or improve survival outright $[28,29]$. The present data do not elucidate whether hypoalbuminaemia represents a marker of poor outcome or a modifiable factor that can be targeted to improve survival in patients with metastatic disease. However, these data do underscore the importance of nutritional status in patient outcomes. Furthermore, our present report should serve as a catalyst for further work to better understand if correction of nutritional status affords a therapeutic opportunity for patients with mRCC.

Similar to other retrospective studies, the present study has limitations. As with all retrospective data, treatment choice was not randomised resulting in the likelihood of selection bias. Specifically, despite controlling for all available clinically relevant and statistically significant covariates, unmeasured confounders as well as parameters not significant on univariate analysis may influence our present results. Indeed, controlling for clinical and pathological factors indicative of tumour stage in the multivariable analysis, hypoalbuminaemia was independently associated with worse outcomes. Nevertheless, patients with hypoalbuminaemia had more advanced disease, which may confound our present findings. Unfortunately, in this dataset where a large number of patients probably travelled a long distance for surgery, data about receipt of systemic therapy and readmission rates were not fully captured limiting our ability to evaluate these factors. Nevertheless, relative strengths include the multi-institutional design, harnessing two prospectively managed databases that are vigorously maintained with robust processes to update vital status. In the absence of rigorously tested consensus criteria to define poor nutritional status in patients before renal surgery, the parameters used in the present study were available in all patients, objective, and easily obtainable from preoperative data and nutritional status variables.

In conclusion, our present data underscore the role of hypoalbuminaemia as an independent risk factor for overall and disease-specific mortality in patients with mRCC undergoing CN. Serum albumin (a readily available, easily measured parameter) when low, identifies patients at increased risk for early mortality and is highly prevalent in patients with advanced RCC. We believe these data and recent data from other institutions would spark interest in determining whether poor nutrition is a modifiable risk or simply a marker of poor prognosis. Certainly, if a trial of targeted therapy is chosen upfront or if surgical intervention is delayed for any reason, dietary intervention to potentially correct hypoalbuminaemia is not likely to harm and may offer benefits.

\section{Source of Funding}

This publication was supported by the National Cancer Institute at the National Institutes of Health (grant number: P30 CA006927, RU) and the Department of Defense, Physician Research Training Award (A.K.). No financial disclosures. 


\section{Conflict of Interests}

No authors have any conflict of interests related to this manuscript to report.

\section{References}

1 Mickisch GH, Garin A, van Poppel H et al. Radical nephrectomy plus interferon-alfa-based immunotherapy compared with interferon alfa alone in metastatic renal-cell carcinoma: a randomised trial. Lancet 2001; 358: 966-70

2 Flanigan RC, Mickisch G, Sylvester R, Tangen C, Van Poppel H, Crawford ED. Cytoreductive nephrectomy in patients with metastatic renal cancer: a combined analysis. J Urol 2004; 171: 1071-6

3 Flanigan RC, Salmon SE, Blumenstein BA et al. Nephrectomy followed by interferon Alfa- $2 \mathrm{~b}$ compared with interferon Alfa- $2 \mathrm{~b}$ alone for metastatic renal-cell cancer. N Engl J Med 2001; 345: 1655-9

4 Motzer RJ, Hutson TE, Tomczak P et al. Overall survival and updated results for sunitinib compared with interferon alfa in patients with metastatic renal cell carcinoma. J Clin Oncol 2009; 27: 3584-90

5 Hudes G, Carducci M, Tomczak P et al. Temsirolimus, interferon alfa, or both for advanced renal-cell carcinoma. N Engl J Med 2007; 356: 2271-81

6 Escudier B, Eisen T, Stadler WM et al. Sorafenib in advanced clear-cell renal-cell carcinoma. N Engl J Med 2007; 356: 125-34

7 Crispen PL, Blute ML. Role of cytoreductive nephrectomy in the era of targeted therapy for renal cell carcinoma. Curr Urol Rep 2012; 13: $38-46$

8 Kutikov A, Uzzo RG, Caraway A et al. Use of systemic therapy and factors affecting survival for patients undergoing cytoreductive nephrectomy. BJU Int 2010; 106: 218-23

9 Culp SH, Tannir NM, Abel EJ et al. Can we better select patients with metastatic renal cell carcinoma for cytoreductive nephrectomy? Cancer 2010; 116: 3378-88

10 O'Malley RL, Brewer KA, Hayn MH et al. Impact of cytoreductive nephrectomy on eligibility for systemic treatment and effects on survival: are surgical complications or disease related factors responsible? Urology 2011; 78: 595-600

11 Trinh QD, Bianchi M, Hansen J et al. In-hospital mortality and failure to rescue after cytoreductive nephrectomy. Eur Urol 2013; 63: 1107-14

12 Kassouf W, Sanchez-Ortiz R, Tamboli P et al. Cytoreductive nephrectomy for T4NxM1 renal cell carcinoma: the M.D. Anderson Cancer Center experience. Urology 2007; 69: 835-8

13 Kelsen DP, Ginsberg R, Pajak TF et al. Chemotherapy followed by surgery compared with surgery alone for localized esophageal cancer. N Engl J Med 1998; 339: 1979-84

14 Garth AK, Newsome CM, Simmance N, Crowe TC. Nutritional status, nutrition practices and post-operative complications in patients with gastrointestinal cancer. J Hum Nutr Diet 2010; 23: 393-401

15 Alves A, Panis Y, Mathieu P et al. Postoperative mortality and morbidity in French patients undergoing colorectal surgery: results of a prospective multicenter study. Arch Surg 2005; 140: 278-84
16 Mullen JT, Davenport DL, Hutter MM et al. Impact of body mass index on perioperative outcomes in patients undergoing major intra-abdominal cancer surgery. Ann Surg Oncol 2008; 15: 2164-72

17 Morgan TM, Tang D, Stratton KL et al. Preoperative nutritional status is an important predictor of survival in patients undergoing surgery for renal cell carcinoma. Eur Urol 2011; 59: 923-8

18 Margulis V, Shariat SF, Rapoport Y et al. Development of accurate models for individualized prediction of survival after cytoreductive nephrectomy for metastatic renal cell carcinoma. Eur Urol 2013; 63: $947-52$

19 National Kidney Foundation, Inc. NKF KDOQI Guidelines, 2002. 2002. Available at: http://www2.kidney.org/professionals/KDOQI/guidelines _ckd/p4_class_g1.htm. Accessed November 2014

20 Edge SB, Joint A. Committee on Cancer. AJCC Cancer Staging Manual, 7th edn. New York: Springer, 2010

21 Bex A, Jonasch E, Kirkali $\mathrm{Z}$ et al. Integrating surgery with targeted therapies for renal cell carcinoma: current evidence and ongoing trials. Eur Urol 2010; 58: 819-28

22 ClinicalTrials.gov. Clinical Trial to Assess the Importance of Nephrectomy (CARMENA). 2009. Available at: http://clinicaltrials.gov/show/ NCT00930033. Accessed October 2014

23 Gustafsson UO, Ljungqvist O. Perioperative nutritional management in digestive tract surgery. Curr Opin Clin Nutr Metab Care 2011; 14: 504-9

24 Fearon KC, Jenkins JT, Carli F, Lassen K. Patient optimization for gastrointestinal cancer surgery. Br J Surg 2013; 100: 15-27

25 Huhmann MB, August DA. Perioperative nutrition support in cancer patients. Nutr Clin Pract 2012; 27: 586-92

26 Gregg JR, Cookson MS, Phillips S et al. Effect of preoperative nutritional deficiency on mortality after radical cystectomy for bladder cancer. J Urol 2011; 185: 90-6

27 Karl A, Rittler P, Buchner A et al. Prospective assessment of malnutrition in urologic patients. Urology 2009; 73: 1072-6

28 Heys SD, Walker LG, Deehan DJ, Eremin OE. Serum albumin: a prognostic indicator in patients with colorectal cancer. J R Coll Surg Edinb 1998; 43: 163-8

29 Veenhof AA, Vlug MS, van der Pas MH et al. Surgical stress response and postoperative immune function after laparoscopy or open surgery with fast track or standard perioperative care: a randomized trial. Ann Surg 2012; 255: 216-21

Correspondence: Alexander Kutikov, MD, Division of Urologic Oncology, Fox Chase Cancer Center-Temple University Health System, 333 Cottman Avenue, Philadelphia, PA 19111, USA.

e-mail: alexander.kutikov@fccc.edu

Abbreviations: BMI, body mass index; CN, cytoreductive nephrectomy; DSS, disease-specific survival; HR, hazard ratio; $\mathrm{IQR}$, interquartile range; OS, overall survival; mRCC, metastatic RCC. 